\title{
Assistência obstétrica/neonatal: ampliação da prática clínica do enfermeiro na Atenção Primária
}

\author{
Obstetric/neonatal care: expansion of nurses' clinical practice in Primary Care
}

\section{Atención obstétrica/neonatal: ampliación de la práctica clínica del enfermero en la atención primaria}

Tamiris Scoz Amorim ${ }^{1}$

Marli Terezinha Stein Backes ${ }^{1}$

Evangelia Kotzias Atherino dos Santos

Kamylla Santos da Cunha

Vânia Sorgatto Collaço

\section{Descritores}

Atenção primária à saúde; Acesso aos serviços de saúde; Autonomia profissional; Cuidados de enfermagem; Enfermagem obstétrica

\section{Keywords}

Primary health care; Health services accessibility; Professional autonomy; Nursing care; Obstetric nursing

\section{Descriptors}

Atención primaria de salud; Accesibilidad a los servicios de salud; Autonomía profesional; Atención de enfermería; Enfermería obstétrica

\section{Submetido}

29 de Agosto de 2018

Aceito

16 de Abril de 2019

\section{Resumo}

Objetivo: Compreender o significado da ampliação da prática clínica do enfermeiro na atenção obstétrica e neonatal no contexto da atenção primária.

Métodos: Pesquisa qualitativa, com o referencial teórico filosófico do Pensamento Complexo de Edgar Morin e com o referencial metodológico da Teoria Fundamentada nos Dados. As etapas de coleta, análise e construcão da teoria ocorreram em sequências alternadas, entre setembro de 2016 a setembro de 2017. A saturação teórica dos dados ocorreu com a inclusão de 11 entrevistas individuais com enfermeiras da atencão primária e com quatro observaç̃es participantes. Os dados foram organizados por meio do software NVIV010® e analisados em três etapas: codificação aberta, axial e integração.

Resultados: Emergiu o fenômeno central "Provocando mudanças na atuação do enfermeiro da atenção primária" e a três categorias: Compreendendo os significados da gestão do cuidado de Enfermagem na atenção primária; lidando com o movimento antagonista e regulador que influencia a qualidade e melhorando a qualidade para promover a resolutividade da atenção primária.

Conclusão: Os significados do movimento de liderança em prol da ampliação da prática clínica do enfermeiro na Atenção Primária relacionam-se com: as resistências na postura de alguns profissionais, a inconstância de recursos humanos e materiais, mas, sobretudo, com a busca pela essência do cuidar.

\section{Abstract}

Objective: To understand the meaning of the expansion of nurses' clinical practice in obstetric and neonatal care in the primary care context. Methods: Qualitative study in which were adopted the theoretical-philosophical reference of Edgar Morin's Complex Thought and the methodological framework of the Grounded Theory. The stages of collection, analysis and construction of the theory occurred in alternating sequences between September 2016 and September 2017. The theoretical saturation of data was reached after including 11 individual interviews with primary care nurses and four participant observations. Data were organized using the NVIV010 ${ }^{\circledR}$ software and analyzed in three steps: open, axial and integration coding.

Results: The central phenomenon "Provoking changes in the performance of primary care nurses" and three categories emerged: Understanding the meanings of nursing care management in primary care; Dealing with the antagonistic and regulating movement that influences quality; and Improving quality to promote the resolution of primary care.

Conclusion: The meanings of the leadership movement in favor of the expansion of nurses' clinical practice in primary care are related to the resistant posture of some professionals, the inconstancy of human and material resources, but above all, the search for the care essence.

\section{Resumen}

Objetivo: comprender el significado de la ampliación de la práctica clínica del enfermero en la atención obstétrica y neonatal en el contexto de la atención primaria.

Métodos: investigación cualitativa, con base teórica filosófica del Pensamiento Complejo de Edgar Morin y base metodológica de la Teoría Fundamentada en los Datos. Las etapas de recolección, análisis y elaboración de la teoría se realizaron en secuencias alternadas, entre septiembre de 2016 y septiembre de 2017. La saturación teórica de los datos se realizó con la inclusión de 11 entrevistas individuales con enfermeras de atención primaria y con 4 observaciones participantes. Los datos fueron organizados por medio del software NVIV010® y analizados en tres etapas: codificación abierta, axial e integración.

Resultados: surgió el fenómeno central "provocar cambios en la actuación del enfermero de la atención primaria" y las tres categorías: comprender los significados de la gestión del cuidado de enfermería en la atención primaria, lidiar con el movimiento antagonista y regulador que influye en la calidad, y mejorar la calidad para promover la resolutividad de la atención primaria.

Conclusión: los significados del movimiento de liderazgo a favor de la ampliación de la práctica clínica del enfermero en la atención primaria se relacionan con las resistencias en la postura de algunos profesionales, la inconstancia de recursos humanos y materiales, pero, sobre todo, con la búsqueda de la esencia del cuidar.

\section{Autor correspondente}

Tamiris Scoz Amorim

https://orcid.org/0000-0003-0220-7526

E-mail: tamiscoz@hotmail.com

\section{DOI}

http://dx.doi.org/10.1590/1982

0194201900050

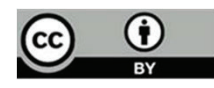

\section{Como citar:}

Amorim TS, Backes MT, Santos EK, Cunha KS, Collaço VS. Assistência obstétrica/neonatal: ampliação da prática clínica do enfermeiro na Atenção Primária. Acta Paul Enferm. 2019;32(4):358-64. 


\section{Introdução}

A atenção obstétrica e neonatal atravessa momentos de significativas transformaçóes. Os desafios contemporâneos convergem para a necessidade de adotar um modelo humanizado de assistência baseado nas boas práticas de atenção à gestação, ao parto e ao nascimento. ${ }^{(1)}$ Atualmente, a dificuldade de acesso aos serviços de saúde é um fator contrário à qualidade da atenção obstétrica. ${ }^{(2)}$ Nessa direção, os(as) enfermeiros(as) atuantes na Atenção Primária à Saúde (APS) representam uma peça chave na proteção e na promoção da saúde da mulher, do concepto, do Recém-Nascido (RN) e do acompanhante/familiar. ${ }^{(3)}$

Os(as) enfermeiros(as) da APS, por meio da gestão do cuidado de enfermagem podem estabelecer um cuidado inovador ao articularem ciência e arte para uma análise crítica da situação. Nesse contexto, a organização dos recursos disponíveis e a avaliação dos cuidados garantem a qualidade dos serviços de saúde. ${ }^{(4)}$

Uma das ferramentas disponíveis aos enfermeiros(as) para realizarem a gestão do cuidado são os protocolos clínicos de enfermagem (PCE). Os PCE, neste cenário, foram criados a partir das necessidades dos(as) enfermeiros(as) da assistência sobre a sua prática clínica, fornecendo subsídios de maneira mais diretiva e prática, baseados nas melhores evidências. Respaldando-os legalmente para as condutas e tomadas de decisão. ${ }^{(5,6)}$ No entanto, a implementação de PCE depara-se com barreiras institucionais, humanas e materiais, repercutindo, principalmente, na autonomia profissional. ${ }^{(5)}$ Igualmente, envolve interaçóes/açóes as quais precisam ser compreendidas para que os PCE ganhem significado para a ampliação da prática clínica de enfermeiros(as) na APS. ${ }^{(3-5)}$

A diminuição da mortalidade materna e infantil relacionada às fragilidades na atenção pré-natal é um desafio para a saúde pública e poderia ser alcançada por meio de melhorias na oferta e no acesso aos serviços de saúde. ${ }^{(2,3)}$ Logo, a importância de compreender as açóes, as barreiras e as inquietaçóes que motivaram um grupo de enfermeiros(as) a tomarem a liderança em prol de mudanças na prática clínica da enfermagem está ligada à capacidade de inspirar novos movimentos em benefício das mulheres, enfermagem e APS.

A realização deste estudo também se justifica pela necessidade de se investigar sobre a temática, uma vez que não foram encontrados estudos específicos relacionados à gestão do cuidado de enfermagem voltada à atenção obstétrica e neonatal por enfermeiros(as) da APS, o que é um tema relevante na realidade brasileira.

Este é um recorte da Dissertação de Mestrado intitulada: "O significado atribuído à gestão do cuidado de enfermagem para a qualidade da atenção obstétrica e neonatal por enfermeiras da Atenção Primária à Saúde de Florianópolis". Este recorte tem por objetivo compreender o significado da ampliação da prática clínica do enfermeiro na atenção obstétrica e neonatal no contexto da atenção primária.

\section{Métodos}

Trata-se de uma pesquisa qualitativa com o referencial teórico-metodológico da Teoria Fundamentada nos Dados (TFD) (vertente Straussiana) e o referencial teórico-filosófico do Pensamento Complexo de Edgar Morin. ${ }^{(7,8)}$

A pesquisa envolveu cinco Centros de Saúde (CS) e a Secretaria Municipal de Saúde (SMS) de Florianópolis/SC/Brasil. A APS deste município conta com $100 \%$ de cobertura das equipes de Estratégia de Saúde da Família (ESF) em seus 50 (cinquenta) CS. Além da implantação do Programa Capital Criança ${ }^{(9)}$ e do Protocolo de Enfermagem da Saúde da Mulher. ${ }^{(10)}$

Foram critérios de inclusão: (1) ser enfermeiro (a) da SMS/Florianópolis (2) ter experiência maior que seis meses na área, (3) ter expertise em gestáo do cuidado, obstetrícia e/ou saúde da mulher.

Utilizou-se como instrumentos de coleta de dados a observação participante, um questionário fechado para identificação da expertise dos sujeitos e a entrevista semi-estruturada, com a questão norteadora: $\mathrm{O}$ que significa para você a gestão do cuidado de Enfermagem para a qualidade da atenção obstétrica e neonatal na APS? 
A coleta de dados ocorreu entre os meses de setembro de 2016 a setembro de 2017. Foram realizadas 11 (onze) entrevistas com enfermeiras da APS, as quais foram contatadas via endereço eletrônico institucional e as entrevistas ocorreram em seus locais de trabalho. Também foi realizado observação participante em 03 (três) cenários diferentes que compreenderam os encontros de um Grupo de Gestantes, Consulta de Enfermagem no pré-natal e reuniâo de enfermeiros(as) da APS. Estas Observaçóes foram registradas em diário de campo.

A análise dos dados seguiu três etapas: codificação aberta, axial e integração. Os códigos emergentes foram sistematicamente agrupados, comparados e ordenados, com apoio do software $\mathrm{NVIVO}^{\oplus} 10$. $\mathrm{Na}$ codificação aberta, a análise estendeu-se aos possíveis informantes e locais. Os códigos emergentes desta análise compuseram categorias e subcategorias. $\mathrm{Na}$ etapa axial, codificam-se os dados em torno da categoria central do estudo. Nesta etapa, utilizaram-se os componentes do Modelo Paradigmático de Corbin e Strauss: Condiçôes, Açôes/interaçôes e Consequências. ${ }^{(6)} \mathrm{Na}$ fase de integração, ocorreu a conexão dos conceitos que sustentam a Teoria construída. As etapas de coleta, análise e construçáo da teoria ocorreram de maneira alternada. A coleta de dados encerrou com a saturação teórica dos dados, determinada pela repetição de informaçóes pelos participantes sobre o fenômeno e ausência de novos elementos relevantes para o objetivo do estudo. ${ }^{(6)}$

Este estudo integra um Macroprojeto de pesquisa que recebeu parecer favorável do Comitê de Ética em Pesquisa por meio do número 1.148.080 e do CAEE: 43112415.5.0000.0121. As informaçóes contidas nos Termos de Consentimento Livre e Esclarecidas (TCLE) foram explicadas para cada participante, antes da assinatura do termo. Manteve-se o sigilo sobre a identidade dos sujeitos com o uso da letra "E" de enfermeira e um numeral oriundo da ordem das entrevistas [E1... E11].

\section{Resultados}

Das 11 (onze) participantes: 05 (cinco) eram enfermeiras da ESF; 03 (três) eram coordenadoras da unidade; 02 (duas) eram gestoras na SMS e 01 (uma) exercia a função de enfermeira assistencial e coordenadora da unidade. A teoria substantiva derivada do processo analítico revela os significados inter-relacionados dos movimentos/interaçóes entre mulher-enfermeira-cuidado de Enfermagem. O fenômeno central da pesquisa: "Provocando mudanças na atuação do enfermeiro da atenção primária”, sustenta 03 (três) categorias: Compreendendo os significados da gestão do cuidado de enfermagem na atenção primária, Lidando com o movimento antagonista e regulador que influencia a qualidade e, Melhorando a qualidade para promover a resolutividade da atenção primária. O Modelo Teórico (Figura 1) apresenta o fenômeno central integrado aos componentes analíticos: Condiçóes, Açóes/interaçóes e Consequências. ${ }^{(6,7)}$

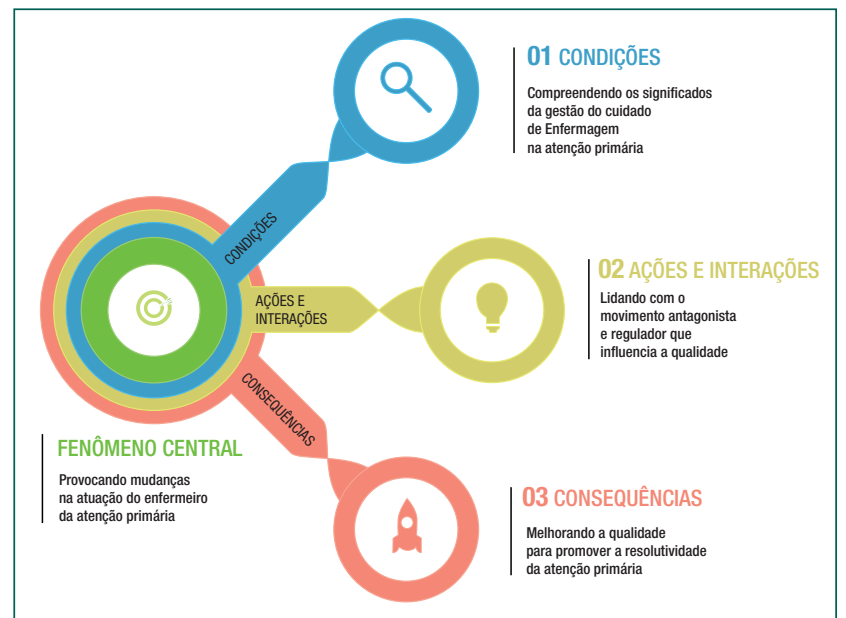

Figura 1. Modelo Teórico "Provocando mudanças na atuação do enfermeiro da atenção primária"

\section{Compreendendo os significados da gestão do cuidado de enfermagem na atenção primária}

A atuação clínica do(a) enfermeiro(a) da APS permaneceu, por muito tempo, limitada às açóes de saúde com baixa resolutividade. Na prática, quando ocorriam situaçóes de saúde em que fosse preciso um manejo clínico, os(as) enfermeiros(as) não contavam com respaldo para atender a demanda de cuidado apresentada pelo indivíduo.

"Achavam que enfermeiro da atenção primária só fazia, basicamente, reunião com o agente de saúde, 
promoção da saúde, no máximo, um pré-natalzinho e um preventivo. [...] Quando chegava a parte de ter um pouco mais de autonomia clinica para o enfermeiro, a gente tinha algumas reservas". E1

Cônscios de que estas contradiçóes sobre a prática clínica do enfermeiro representam um entrave para a qualidade da atenção, alguns(mas) enfermeiros(as) da APS assumiram o protagonismo de iniciar um movimento de discussão sobre PCE, culminando na criação da Comissão Permanente de Sistematização da Assistência de Enfermagem. Além de ter implantado a Sistematização da Assistência de Enfermagem (SAE), a comissão têm por objetivos: discutir a resolutividade da enfermagem e estabelecer melhorias no manejo clínico da atenção à saúde dos usuários da APS.

Para dar vida a esse movimento, foi necessário elaborar os PCE e implantar o Guia de Práticas Clínicas da Atenção Primária (PACK), dois instrumentos de gestão do cuidado voltados para enfermeiros e médicos para a tomada de decisão sobre as condutas clínicas de atenção ao pré-natal, puerpério e período neonatal. A condição essencial, apontada pelas entrevistadas, para a ampliação da prática clínica deu-se pela compreensáo de que o processo de trabalho na APS tem caráter multidisciplinar, interdisciplinar, intersetorial e integral. Notou-se que era preciso discutir a atuação do enfermeiro neste contexto. Esse movimento de liderança encontrou barreiras por parte de médicos e enfermeiros(as) que se posicionaram contrários à proposta e, de outros, que manifestaram apoio.

"Tem colegas médicos que nos apoiam bastante e tem colegas médicos que nos olham meio atravessadinhos, ainda. É normal, a gente está rompendo um método tradicional que é mais voltado para o médico e a gente está agora voltando o nosso olhar para a equipe mesmo. O que a equipe pode resolver. $O$ que o enfermeiro pode resolver. $E$ isso é excelente!" E4

"Tinha quem dizia [enfermeiros] que não ia abrir Protocolo nenhum. Que isso era trabalho do médico! Mas, a enfermagem não precisa tomar a benção com nenhuma categoria para fazer. Nós assumimos o protagonismo de fazer para a enfermagem”. E1
Independente de contar com o apoio dos colegas, é importante que a Enfermagem compreenda que não precisa do consentimento de outras categorias profissionais para estar atuando no cenário de cuidados.

\section{Lidando com o movimento antagonista e regulador que influencia a qualidade}

A falta de uma equipe de ESF em número suficiente, o aumento da demanda de trabalho, a insuficiência de insumos assistenciais, as limitaçóes do espaço físico do CS para a realização de Grupos de Gestantes, o momento de crise e instabilidade da Saúde Pública foram referidos, pelas participantes, como movimentos contrários à qualidade da atenção obstétrica e neonatal. No entanto, nota-se muita vontade de trabalhar e fazer um serviço de qualidade.

"As pessoas estão cansadas de falta de médicos, e falta disso e daquilo, mas mesmo assim querem fazer mudar". E5

A falta de comunicação entre os serviços de saúde apareceu como uma fragilidade no trabalho em Rede de Atenção à Saúde (RAS). As informaçôes sobre os cuidados na maternidade são conhecidas por meio do relato da mãe e dos registros na Caderneta da Gestante e da Criança. Foi referido como positivo ter o Programa Capital Criança, como forma de garantir o retorno agendado para as consultas de puericultura no CS. Para melhor lidar com esses entraves, pactuaram-se açôes/interaçóes como: a reflexão sobre a visão holística da enfermagem, ou seja, a capacidade de oferecer um cuidado integral e resolutivo para a mulher/família, a solidariedade entre a equipe, a interconsulta entre médico e enfermeira e a discussão em Equipe.

\section{Melhorando a qualidade para promover a resolutividade da atenção primária}

A partir da implantação dos PCE e do PACK houve o fortalecimento da competência clínica do enfermeiro. Aquele profissional que, no início, mostrou resistência em mudar seu processo de trabalho passou a empoderar-se e a sentir-se importante no contexto assistencial, e isso repercutiu na resolutivi- 
dade da enfermagem e na ampliação do acesso das usuárias.

"Um protocolo voltado para o enfermeiro se empoderar cada vez mais e ter qualidade no seu atendimento. Isso repercute diretamente na gestão do cuidado em obstetrícia”. E4

"Esse [Protocolo] deu uma incrementada e ajuda porque tem a questão da resolutividade, principalmente, nessa questão de medicamentos e solicitação de exames". E3

As usuárias, em contrapartida, respondem, com preferência, pela consulta de enfermagem pré-natal realizada pelo(a) enfermeiro(a) e com o sentimento de segurança, ligado ao vínculo, ao afeto e ao cuidado dos(as) enfermeiros(as), acompanhado pela promoção do empoderamento e da autonomia do casal grávido.

"Os pacientes preferem consultar com o enfermeiro porque a gente já tem uma autonomia para a questão de medicamentos, pedir exames. Então assim, o atendimento da enfermagem tem um tempo muito maior, é mais caloroso, afetuoso!" E8

"O enfermeiro é o Severino Quebra Galho. O enfermeiro está na Portaria. A gente está pregando coisa na parede. A gente precisa fazer... Ai acaba se perdendo um pouco pelo acúmulo de funçóes que a gente tem". E9

Nesse sentido, as entrevistadas compartilharam sentimentos ambíguos de satisfação profissional com o atendimento oferecido, tanto por gostarem de atender mulheres-gestantes/puérperas/recémnascidos, quanto por perceberem o impacto positivo do cuidado prestado na vida das usuárias. Por outro lado, sentiram que precisavam de estratégias para contornar a sobrecarga de trabalho.

\section{Discussão}

Este estudo, baseado na TFD, ${ }^{(7)}$ revela os significados da vivência de enfermeiras na gestão do cuidado de Enfermagem protagonizada pela liderança de um coletivo interessado em melhorar a qualidade da assistência da APS. A instituição de PCE e a implantação da SAE fortaleceram e ampliaram a prática clínica profissional, consequentemente, melhorou a resolutividade da enfermagem e ampliou o acesso das usuárias.

Desde 1997, com a implantação do Programa Capital Criança, iniciaram-se os primeiros PCE voltados às mulheres e crianças. ${ }^{(9)} \mathrm{O}$ Programa citado pelas entrevistadas é uma referência no município por organizar ações de proteção, promoção e recuperação da saúde de gestantes, puérperas, recém-nascidos e crianças com os CS. Umas delas é a visita de Agentes Educadoras às mães, ainda na maternidade (públicas e privadas), para receber as imunizaçóes e proceder ao agendamento das consultas da mulher e do RN, entre o quinto e o sétimo dia de vida do $\mathrm{RN}$ e a consulta com o dentista para o $30^{\circ}$ dia de vida do RN, no CS. ${ }^{(9)}$

O Protocolo de Atenção à Saúde da Mulher, publicado em 2010 e atualizado constantemente desde 2016, é um documento de suma importância para o processo de trabalho do enfermeiro. Destaca-se aqui que, a elaboração e atualização dos PCE estão baseadas em evidências científicas e garantem a autonomia profissional para o acompanhamento clínico da mulher-gestante de risco habitual, RN e criança. Por meio dos PCE o enfermeiro solicita os exames de pré-natal (de risco habitual), prescreve medicaçóes (quando necessário) e os cuidados de enfermagem, segundo um rol de intervençóes e Diagnósticos de Enfermagem. Para tanto, fundamenta-se na SAE para subsidiar o planejamento, a organização, a coordenação, a avaliação e a execução dos cuidados de Enfermagem. ${ }^{(10)}$

A ampliação da prática clínica de enfermeiros(as), em outro contexto, impulsionou a qualidade da atenção à saúde da população, reduziu custos assistenciais, favoreceu o reconhecimento profissional e melhorou o acesso de usuários aos sistemas de saúde. ${ }^{(1)} \mathrm{O}$ fortalecimento da atenção pré-natal, seguindo critérios de segurança e resolutividade, favoreceu a adesão ao acompanhamento pré-natal. ${ }^{(11,12)}$ Isto é, especialmente, importante para manter o suporte contínuo oferecido pelo 
enfermeiro, mediante o vínculo longitudinal construído na medida em que se realiza um cuidado de enfermagem centrado na mulher, em seu contexto social. ${ }^{(12-15)}$

A sensação de não sentir-se capacitado ou de ter a autonomia profissional limitada apresenta-se como um desafio para a gestão do cuidado de enfermagem. Assim como, o enfrentamento de problemas com a comunicação entre os serviços de saúde e as equipes assistenciais, a burocracia nos serviços e a falta de recursos humanos em número adequado. ${ }^{(13,15-17)}$

A literatura recomenda a construção do vínculo entre mulher-enfermeira, o cuidado de enfermagem focado no contexto social, a ampliação da prática clínica do enfermeiro por meio de PCE e da SAE, como fatores importantes para aumentar a qualidade da atenção à saúde. ${ }^{(2,13-16)}$ Mesmo que a implantação de PCE apresente benefícios, a gestão municipal deve oferecer recursos humanos e materiais suficientes para a proposta de ampliação, caso contrário, pode acarretar em sobrecarga de trabalho para os(as) enfermeiros(as). ${ }^{(14-17)}$

Os PCE caminham junto à iniciativa inédita no Brasil de implantação do Practical Approach To Care Kit (PACK). Este Guia é um modelo de cuidados da África do Sul e apresenta recomendaçóes para 40 (quarenta) sintomas e 20 (vinte) condiçóes crônicas atendidas na APS por enfermeiros e médicos, embasadas nas melhores evidências científicas disponíveis. Conjuntamente com a Universidade da Cidade do Cabo - África do Sul e com o apoio da British Medical Journal do Brasil (BMJ) seu conteúdo foi adaptado à realidade local pelos profissionais de saúde da APS. ${ }^{(18,19)}$

Retomando ao Modelo Teórico, este deve ser compreendido à luz do Pensamento Complexo, ou seja, para além de sua estrutura. Os resultados não podem ser compreendidos como causa-efeito. As interações presentes em um movimento de liderança cultivam traços em constante evolução e incompletude. A cada ação/interação ocorre uma reorganização na atenção e nos envolvidos, por meio dos princípios da autonomia e da morfogênese, para acolher as transformaçóes sociais e das práticas. ${ }^{(7,8)}$

Embora as condiçóes/contradiçóes do processo de trabalho dos enfermeiros(as) sejam produzidas pelo cenário cultural, social e econômico, existem açôes/interaçôes que são os componentes móveis dessa dinâmica. Em retroação, estas entradas e saídas de informaçóes, modulam a busca por outras açôes/interações necessárias para alcançar um objetivo maior. Em consequência, ocorrerá a variação de respostas para cada condição e ação/interação, empenhada no processo. ${ }^{(7,8)}$

\section{Agradecimentos}

Ao Conselho Nacional de Desenvolvimento Científico e Tecnológico (CNPq) pelo financiamento do Macroprojeto de pesquisa coordenado por Marli Terezinha Stein Backes e à Coordenação de Aperfeiçoamento de Pessoal de Nível Superior (CAPES) pela concessão da bolsa de mestrado para Tamiris Scoz Amorim.

\section{Colaborações}

Amorim TS, Backes MTS, Santos EKA, Cunha KS e Collaço VS declaram que contribuíram com a concepção do projeto, análise e interpretação dos dados, redação do artigo, análise e interpretação dos dados e aprovação da versão final a ser publicada.

\section{Conclusão}

Resgatando o objetivo do estudo compreende-se que os significados de um movimento de liderança em prol da ampliação da prática clínica dos(as) enfermeiros(as) da APS relacionam-se com as resistências na postura de alguns profissionais, a inconstância de recursos materiais e humanos, mas, sobretudo, na busca pela essência do cuidar. Embora venham movimentos contrários a estas açóes/interaçóes, cabe aos(às) enfermeiros(as) e à gestão municipal, compreender o aspecto de liderança da enfermagem, focando na resolutividade da APS, no fortalecimento das equipes de ESF e na abertura do acesso às usuárias. Mesmo que existam tensóes e contradiçóes, deve-se perseverar na elaboração de condutas clíni- 
cas, sabendo que podem originar um antagonismo inicial, mas que, paradoxalmente, regulam a ação maior de fortalecimento da enfermagem. Os benefícios decorrentes desta ampliação da prática clínica do enfermeiro para as usuárias/acompanhantes foram: a ampliação do acesso aos serviços de saúde e o empoderamento materno. Para a enfermagem: melhora na resolutividade e na autonomia profissional, com repercussóes positivas para a assistência obstétrica/neonatal na APS. Dessa maneira, é importante realizar outros estudos sobre o tema. Este estudo apresenta como fortaleza o fato de ter sido realizado em um município que atualmente serve como modelo para o país. Como limitação, cita-se o fato de não se poder generalizar estes dados para o país como um todo.

\section{Referências}

1. Sousa AM, Souza KV, Rezende EM, Martins EF, Campos D, Lansky $S$. Practices in childbirth care in maternity with inclusion of obstetric nurses in Belo Horizonte, Minas Gerais. Esc Anna Nery. 2016;20(2):324-31

2. Guimaraes WS, Parente RC, Guimarães TL, Garnelo L. [Access to prenatal care and quality of care in the Family Health Strategy: infrastructure, care, and management]. Cad. Saúde Pública. 2018;34(5):e00110417. Portuguese.

3. Luz LA, Aquino R, Medina MG. [Evaluation of the quality of prenatal care in Brazil]. Saúde Debate. 2018;42(spe2):111-26. Portuguese.

4. Zarate Grajales RA. Management of nursing care. Index Enferm [Intenet]. 2004[cited 2017 jan 18];13(44-45):42-6. Available from: http://scielo.isciii.es/scielo.php?script=sci_arttext\&pid=S1132$12962004000100009 \&$ lng=es. Spanish.

5. Souza MG, Mandu ENT, Elias AN. Perceptions of nurses regarding their work in the family health strategy. Texto contexto enferm [Internet]. 2013[cited 2018 nov 20];22(3):772-9. doi: 10.1590/ S0104-07072013000300025.

6. Kahl C, Meirelles BHS, Lanzoni GMM, Koerich C, Cunha KS. Actions and interactions in clinical nursing practice in Primary Health Care. Rev Esc Enferm USP [Internet]. 2018[cited 2018 dez 16];52:e03327. doi: 10.1590/S1980-220X2017025503327.
7. Corbin J, Strauss A. Basics of qualitative research: techniques and procedures for developing Grounded Theory. Califórnia: SAGE, 2015.

8. Morin E. 0 método: natureza da natureza. Porto Alegre: Sulina, 2016. 477p.

9. Coelho RCAH, Santos EKA, Tavares AM, Tobias LT, Junior RF. Programa Capital Criança Resgatando a cidadania. Florianópolis: Insular, 2004. 192p.

10. Secretaria Municipal de Saúde de Florianópolis. Protocolo de Enfermagem - Saúde da Mulher: Acolhimento às demandas da mulher nos diferentes ciclos de vida. Florianópolis, dezembro de 2016[citado 2018 jan 25]. Versão 1.2. [online] atualizado em setembro de 2017:190. Disponível em: http://www.pmf.sc.gov.br/arquivos/arquivos/ pdf/14_09_2017_15.14.09.259fa5a81e020c350f433fd673d6a319.pdf.

11. Toso BRGO, Filippon J, Giovanella L. Nurses' performance on primary care in the National Health Service in England. Rev Bras Enferm [Internet]. 2016[cited 2017 dez 16];69(1):169-77. doi: 10.1590/00347167.2016690124i.

12. Bortoli CFC, Bisognin P, Wilhelm LA, Prates LA, Sehnem GD, Ressel LB. Factors that enable the performance of nurses in prenatal. Rev Fun Care Online. 2017[cited 2018 jun 16];9(4):978-83. doi: 10.9789/2175-5361.2017.v9i4.978-983.

13. Crossman S, Pfeil M, Moore J, Howe A. A case study exploring employment factors affecting general practice nurse role development. Prim Health Care Res Dev. 2016[cited 2018 jun 16];17:87-97. doi: 10.1017/S1463423615000304.

14. Barbieri-Figueiredo MCA. [Family-centered care: from discourse to practice] [editorial]. Acta Paul Enferm. [Internet]. 2015; [cited 2017 jun 20];28(6):III-IV. doi: 10.1590/1982-0194201500083. Portuguese.

15. Diniz IA, Cavalcante RB, Otoni A, Mata LRF. Perception of primary healthcare management nurses on the nursing process. Rev Bras Enferm. [Internet]. 2015[cited 2017 jun 25];68(2):182-9. doi: 10.1590/0034-7167.2015680204i.

16. Pino JV, Barquero A, Carreño ME. [Gestión del cuidado: valoración y conocimiento de enfermeros(as) de un hospital de la región metropolitana, Chile]. Cienc. enferm. [Internet]. 2015[cited 2017 jun 25];21(1):12742. doi: 10.4067/S0717-95532015000100012. Spanish.

17. Farah BF, Dutra HS, Ramos ACTM, Friedrich DBC. Nurses' perceptions on nursing supervision in Primary Health Care. Rev Rene. 2016[cited 2018 jun 20];17(6): 804-11. doi: 10.15253/21756783.2016000600011.

18. Cornick R, Picken S, Wattrus C, Awotiwon A, Carkeek E, Hannington $\mathrm{J}$, et al. The Practical Approach to Care Kit (PACK) guide: developing a clinical decision support tool to simplify, standardise and strengthen primary healthcare delivery. BMJ Glob Health. 2018[cited 2018 dez 18];3:e000962. doi:10.1136/bmjgh-2018-000962.

19. Wattrus C, Zepeda J, Cornick RV, Zonta R, Andrade MP, Fairall L, et al. Using a mentorship model to localise the Practical Approach to Care Kit (PACK): from South Africa to Brazil. BMJ Glob Health. 2018[cited 2018 dez 18];3:e001016. doi:10.1136/bmjgh-2018-001016. 